\title{
Desarrollo reproductivo del "amancay" Ismene amancaes (Amaryllidaceae) en su ambiente natural
}

\section{Reproductive development of "amancay" Ismene amancaes (Amaryllidaceae) in its natural environment}

\author{
Mery L. Suni, Edisson Pascual, Enoc Jara
}

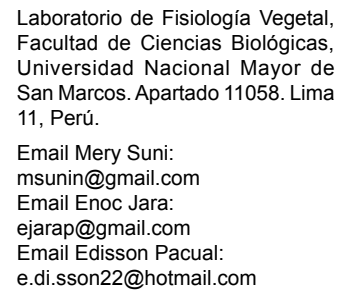

e.di.sson22@hotmail.com

Presentado: $\quad 21 / 05 / 2011$ Aceptado: $\quad$ 20/11/2011 Publicado online: 08/02/2012

\begin{abstract}
Resumen
Ismene amancaes "amancay" es una especie bulbosa característica de las formaciones vegetales denominadas "Lomas" de la costa central del Perú. Emerge al iniciar el periodo de neblina que ocurre en junio, durante el invierno. Presenta flores grandes amarillas y con agradable aroma, muy apreciadas y de valor ornamental. A fin de conocer el desarrollo reproductivo de Ismene amancaes en su ambiente natural se hicieron muestreos mensuales de sus bulbos durante todo un año. Se realizaron observaciones del interior del bulbo para determinar el inicio de la formación y desarrollo de las yemas florales y se relacionó con la formación de sus hojas y la humedad edáfica. Se puede indicar que las primeras yemas florales se hacen evidentes el año anterior a su emergencia, en el mes de diciembre, alcanzando el máximo número de yemas florales en febrero (periodo de verano). La diferenciación de las yemas florales se inicia luego de haberse formado las hojas que saldrán el siguiente año y en el periodo de máximo descenso de la humedad edáfica y de incremento de la temperatura (noviembre). La inflorescencia es la única ramificación que se forma mientras que la yema apical continua formando hojitas. En junio, la pequeña inflorescencia alcanza el cuello del bulbo y avanza seguido por las hojas formadas antes de la inflorescencia siendo envolventes a la inflorescencia misma y a la yema foliar apical. La yema foliar continuará su desarrollo y en julio dos de sus hojas salen del bulbo, las siguientes aun pequeñas quedan dentro y brotarán en el periodo de Lomas del siguiente año. Se puede señalar que el éxito reproductivo de Ismene amancaes en su etapa inicial es dependiente de los fotoasimilados acumulados como biomasa del bulbo en el periodo de Lomas anterior.
\end{abstract}

Palabras clave: Santuario de Amancay, Lima, Desierto costero peruano, bulbo, Lomas

\section{Abstract}

Ismene amancaes "amancay" is a bulbose species typical of the central coast vegetation of Peru called "lomas". This species sprouts in June during the beginning of the winter-fog period. It has large yellow, aromatic flowers valued for their ornamental value. Our goal was to examine the reproductive development of Ismene amancaes in its natural environment, and we recorded monthly observations during a yearlong study. Observations of the interior of the bulbs allowed recording of the beginning of floral bud formation and development, relating them to leaf formation and edaphic humidity. We found that the first floral buds develop the year before their emergence in December, reaching a maximum number of floral buds in February, during the Summer. Floral bud differentiation starts after leaves that will emerge the following year have developed. This occurs during a period of maximum decrease in edaphic humidity and an increase in air temperature (November). The inflorescence is the only branching that develops while the apical bud continues developing leaves. In June, the small inflorescence reaches the neck of the bulb and surpasses it followed by those leaves developed before the inflorescence that surround both the inflorescence and the apical foliar bud. The foliar bud will continue its development, and in July two of the leaves expand, while the smaller ones remain inside the bulb until the following year's Lomas season. It can be noted that the reproductive success of Ismene amancaes in its initial development depends on the photoreserves accumulated in the bulb the previous growth period.

Keywords: Amancay Sanctuary, Lima, Peruvian Coastal Desert, bulb, Lomas.

\section{Introducción}

Ismene amancaes (Ruiz \& Pav) Herb. "amancay" es una planta bulbosa que se desarrolla en la costa central de Perú y es un componente característico del ecosistema de Lomas. Su desarrollo es dependiente de la humedad ambiental que se presenta en los meses de invierno. Es una especie cuya inflorescencia emerge con el inicio del periodo de Lomas seguido del desarrollo foliar, floración, fructificación, producción de semillas y germinación hasta el desarrollo del bulbillo que ocurre al final del periodo de humedad.

La flor de I. amancaes, es apreciada por su color, buen tamaño, forma y fragancia. Su único medio de propagación natural es por semilla, produciendo hasta 13 semillas por flor y 7 flores por planta (dependiendo del tamańo del bulbo) (Agüero \& Suni 1999). Cambios climáticos como los ocasionados por el evento El Niño afectan negativamente los procesos de floración y fructificación, disminuyendo su tasa de propagación. Se estima que sólo el 30\% de las plantas florecen bajo estas condiciones (Agüero \& Suni 1999). También la actividad extractiva del poblador de las zonas aledańas a las Lomas o los visitantes que las recolectan, así como el pastoreo y la expansión de las zonas urbanas y agrícolas, han ocasionado en los últimos años la disminución de su área de distribución y número de individuos en su medio ambiente natural, lo cual ha llevado a considerarla como una especie vulnerable (León et al. 2006).

Pese a su importancia y situación de amenaza son escasos los estudios realizados en esta especie. Es por ello que en el presente trabajo examinamos el desarrollo reproductivo de I. amancaes "amancay" en las Lomas del Santuario de Amancay, Pachacámac (Lima) y evaluamos cómo la temperatura y disponibilidad de agua en el suelo afectan su ciclo de vida. El conocimiento de las condiciones de temperatura y humedad de almacenamiento de los bulbos permitiran el uso y manejo I. amancaes como planta ornamental. 


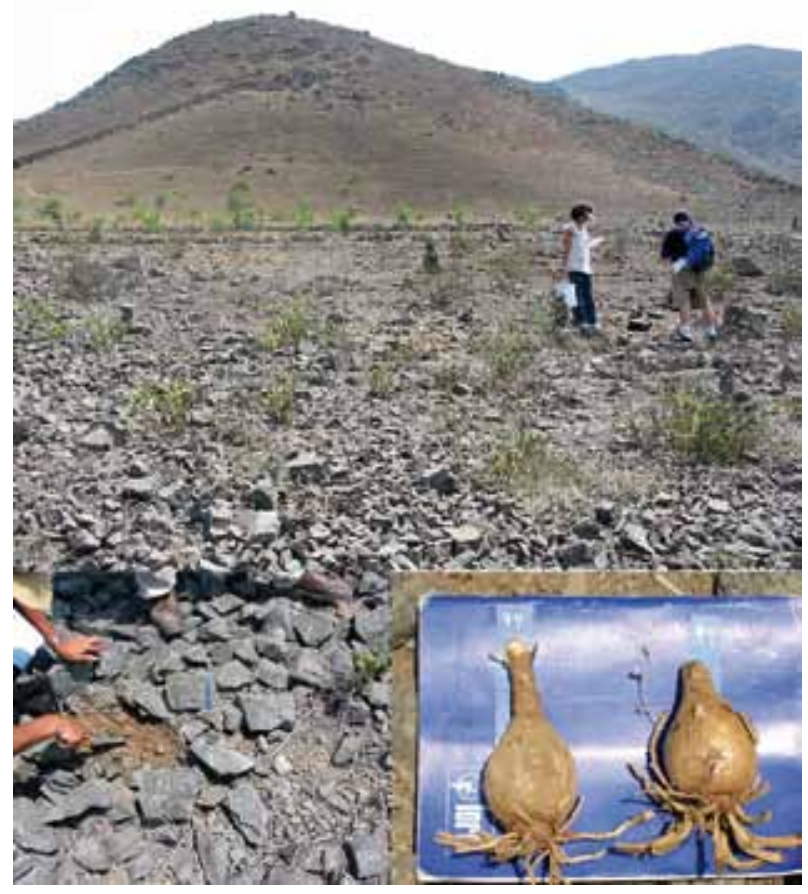

Figura 1. Área de estudio. Vista de una de las parcelas de estudio (30 de enero del 2004). Nótese la ausencia aparente de amancaes y en detalle las características del bulbo extraído y del suelo donde fue ubicado.

\section{Área de estudio}

A fines de octubre del 2003, en la etapa de senescencia foliar de Ismene amancaes, se delimitaron tres parcelas de estudio de $4 \times 5 \mathrm{~m}^{2}$ dentro del Santuario de Amancay, Pachacámac, Lima. Se marcó con estacas la ubicación de 20 bulbos por parcela que presentaban de 5 a 9 hojas (el número de hojas tiene relación con el desarrollo del bulbo y su madurez reproductiva, Suni, M., pers. obs.). Este marcado permitió ubicar los bulbos y realizar los muestreos en el periodo sin desarrollo de la parte aérea (9 meses) (Fig. 1).

\section{Material y métodos}

De enero del 2004 a enero del 2005 se realizaron muestreos mensuales de bulbos y suelo por un total de 13 muestreos.

En cada fecha se retiraron de cada parcela 2 bulbos marcados, material que fue transportado al laboratorio para su posterior análisis. Se registró el peso y características internas del bulbo observando la presencia o ausencia de las estructuras reproductivas así como observaciones sobre la formación de hojas y flores.

Para la medición de la humedad edáfica, mensualmente se colectó una muestra de suelo de cada una de las tres parcelas a los $20 \mathrm{~cm}$ de profundidad, las que se introdujeron inmediatamente a tubos plásticos herméticos y rotulados para su transporte al laboratorio donde fueron pesadas y secadas a $110^{\circ} \mathrm{C}$ hasta peso constante. El porcentaje de humedad (porcentaje de humedad equivalente) fue determinado por diferencia entre el peso húmedo y peso seco dividido entre el peso seco de la muestra del suelo, multiplicado por 100 .

\section{Resultados}

\section{Caracterización del desarrollo de las estructuras repro-} ductivas

En enero de 2004 se observó en el interior hacia la base del bulbo la presencia de 1 a 2 yemas florales muy pequeñas $(3 \mathrm{~mm})$. Este número de yemas en la pequeña inflorescencia se incrementa en el mes de febrero, después de lo cual se mantiene constante (4 yemas en promedio para el material evaluado) hasta el periodo de Lomas en que emergen y se inicia la floración. La pequeña inflorescencia va incrementando su longitud gradualmente (Figs. 2, 3) hasta marzo para posteriormente elongarse

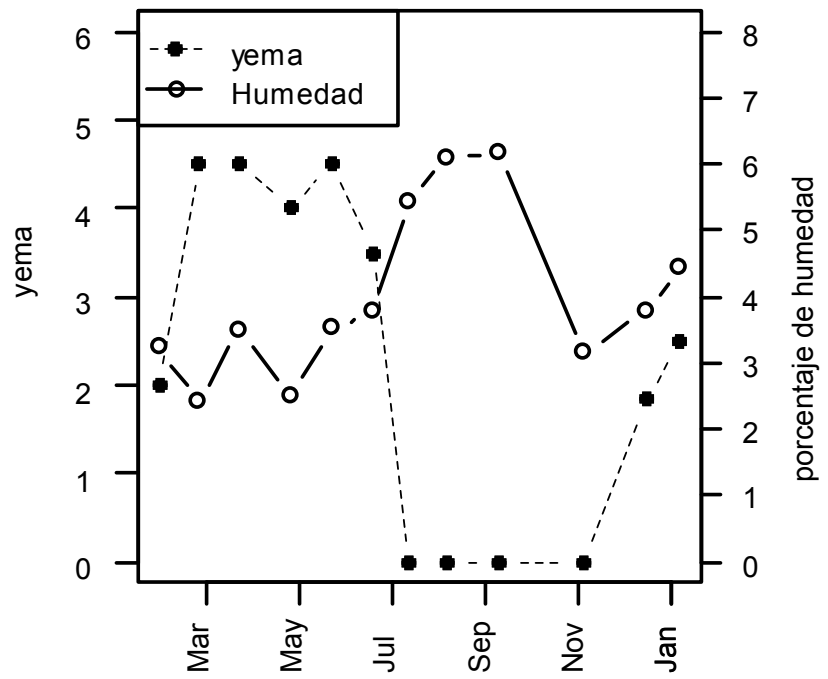

Figura 2. Variación del número de yemas florales en el curso del año y su correlación negativa significativa con el contenido de humedad del suelo.

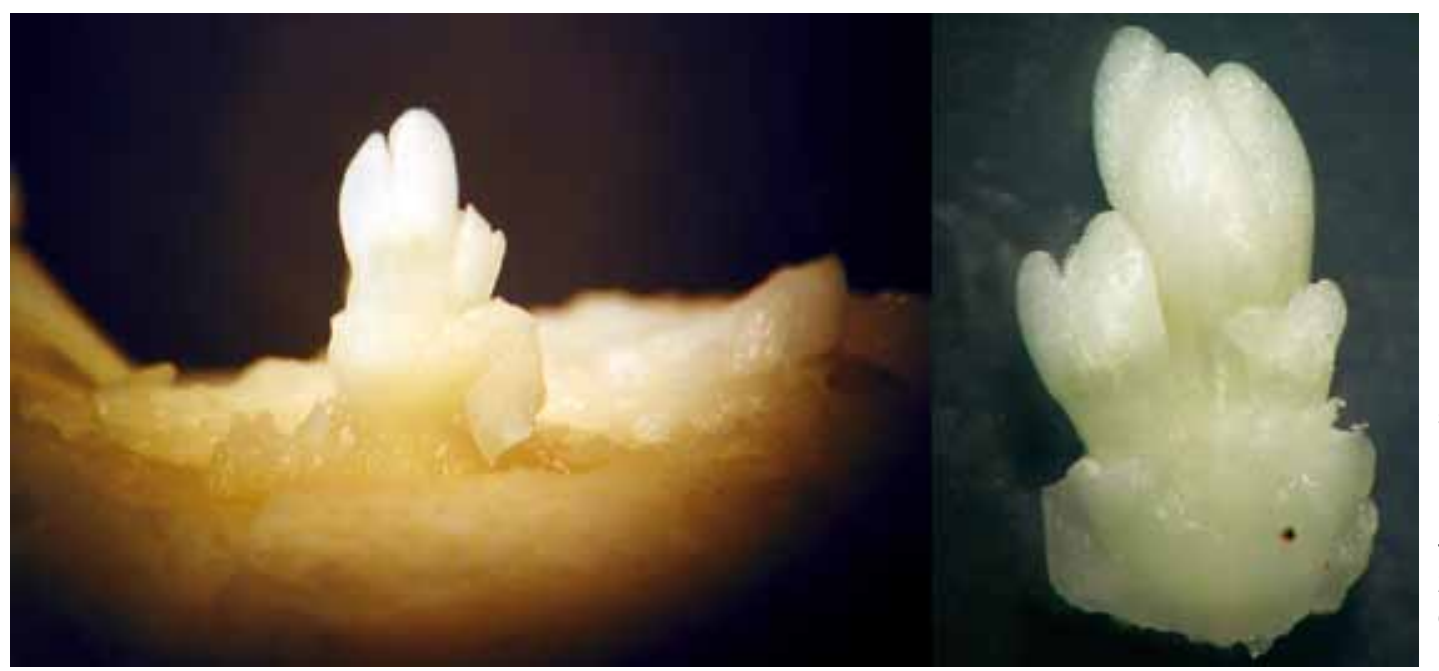

Figura 3. Vistas del desarrollo de la pequeña inflorescencia con algunas yemas florales, en el interior de bulbos colectados en diciembre del 2004 (izquierda) y enero del 2005 (derecha). 


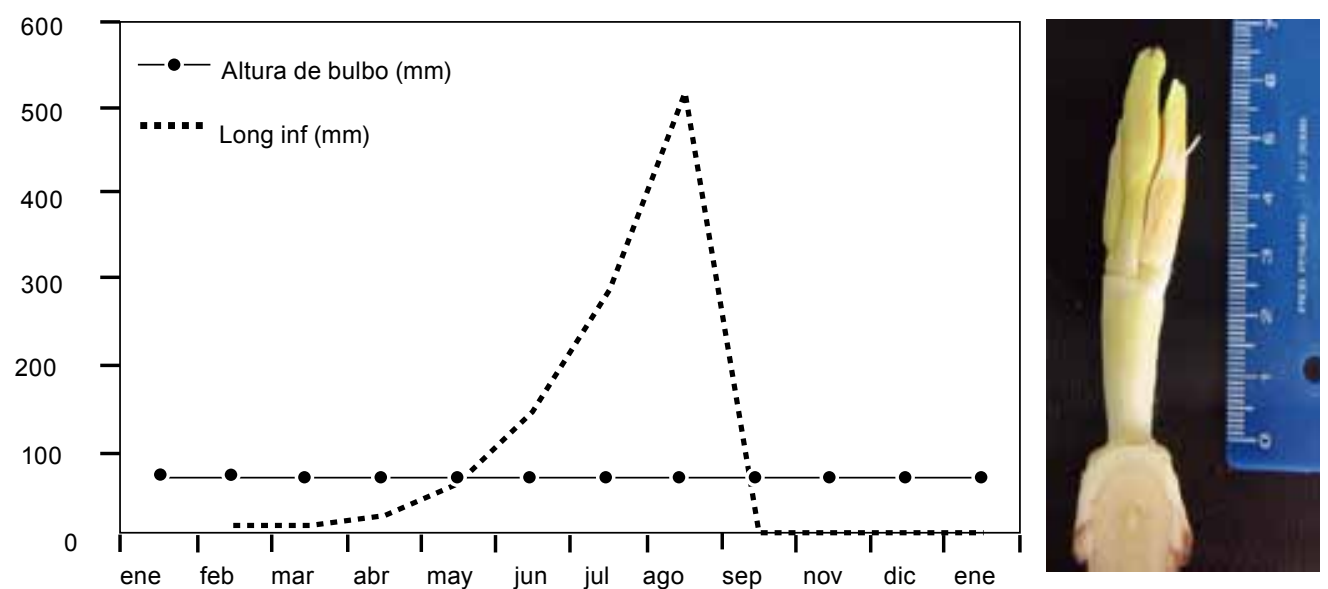

Figura 4. Variación de la longitud de la pequeña inflorescencia en el interior del bulbo de Ismene amancaes en relación a la altura del bulbo. Nótese que para mayo, cuando la tasa de crecimiento se incrementa, alcanza la altura del cuello del bulbo, para continuar en los meses de julio y agosto con un crecimiento exponencial. Vista corresponde a la inflorescencia presente en el interior del bulbo en mayo del 2004.

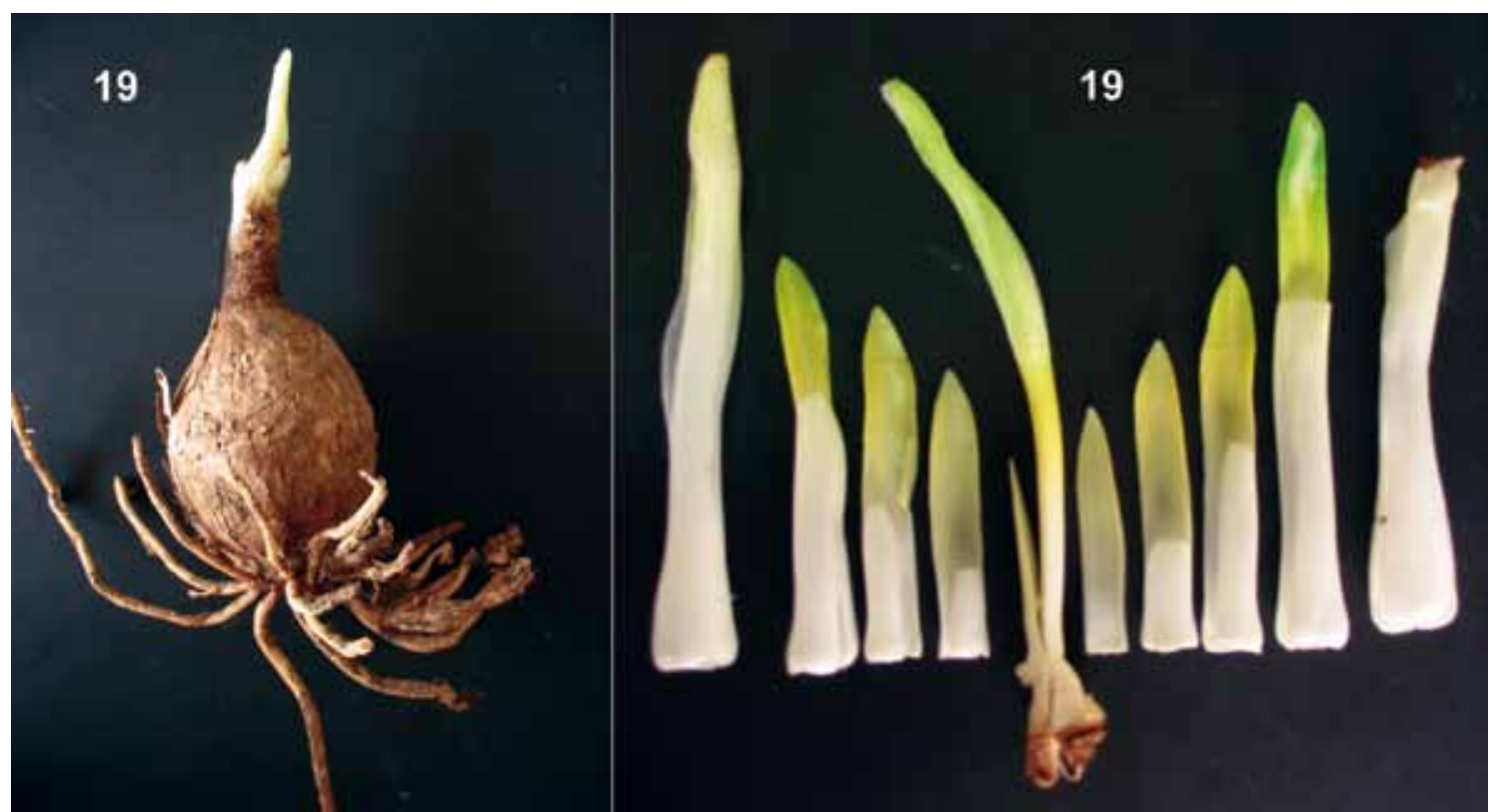

Figura 5. Características del desarrollo de los órganos foliares y florales en el interior del bulbo de Ismene amancaes en junio del 2004 , en la parte central sobre el disco basal se observa la inflorescencia y la yema foliar. Nótese la apariencia del bulbo antes de su disección (foto de la izquierda). Asimismo se muestra las pequeñas hojas desplegadas a ambos lados según su posición en el bulbo.

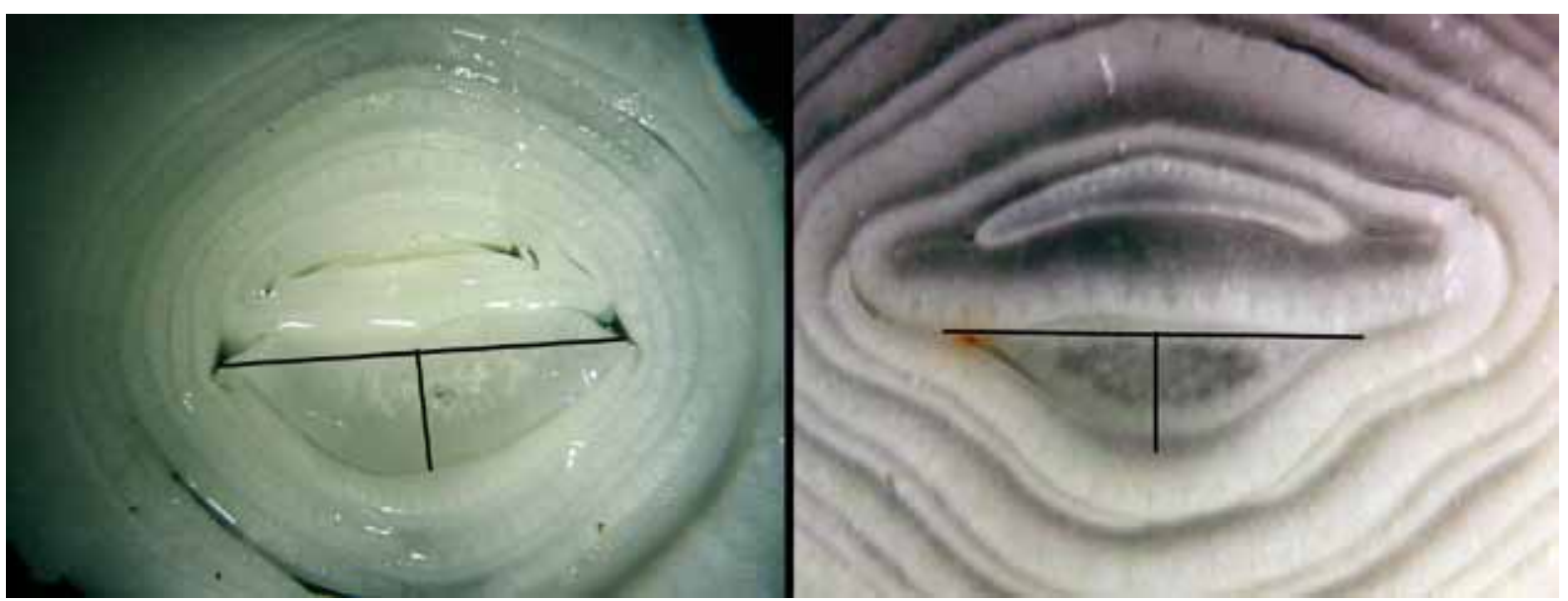

Figura 6. Comparación del tamaño del escapo en corte transversal a nivel medio del bulbo en julio (izquierda) y septiembre (derecha) del 2004. Nótese la reducción de sus dimensiones (véase las líneas). 

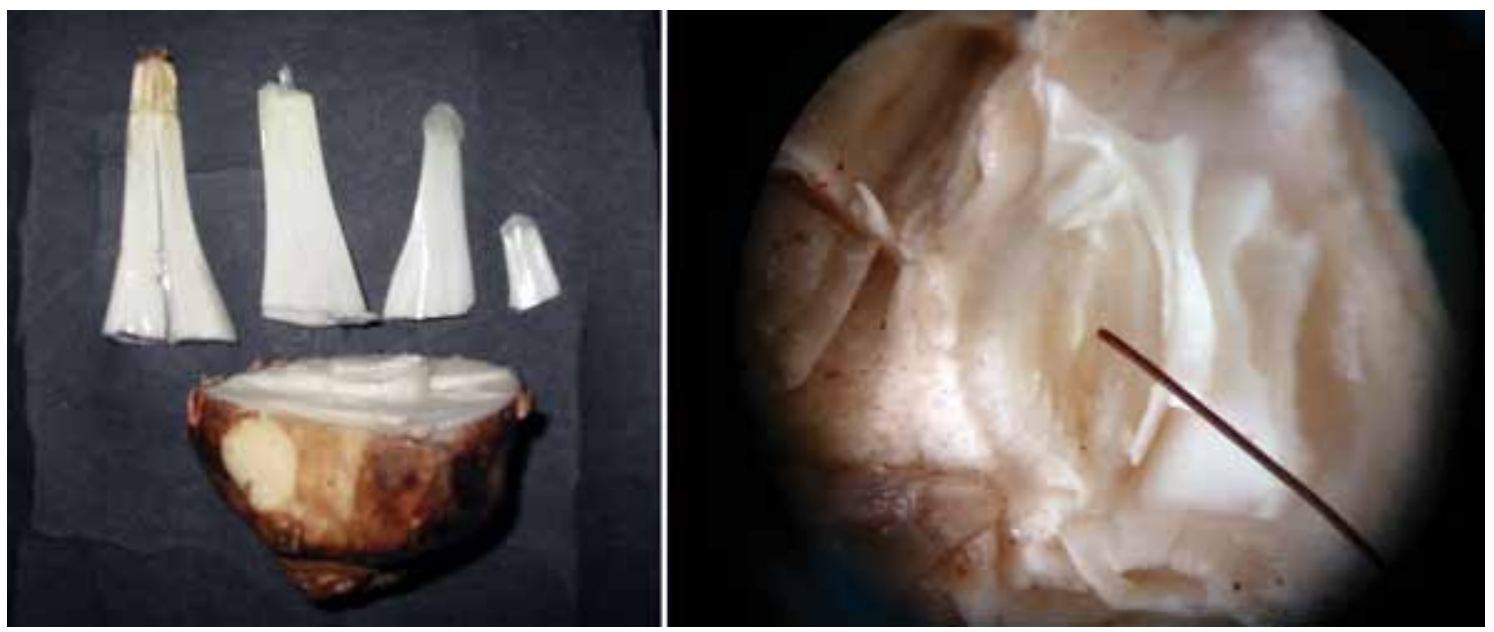

Figura 7. Características del interior del bulbo (foto izquierda), observado en noviembre 2004, nótese la presencia de una hojita (vaina y lámina). Se señala con el puntero el meristemo apical de la yema foliar (visto al microscopio estereoscópico 80X), no fue evidente la presencia de estructuras reproductivas.
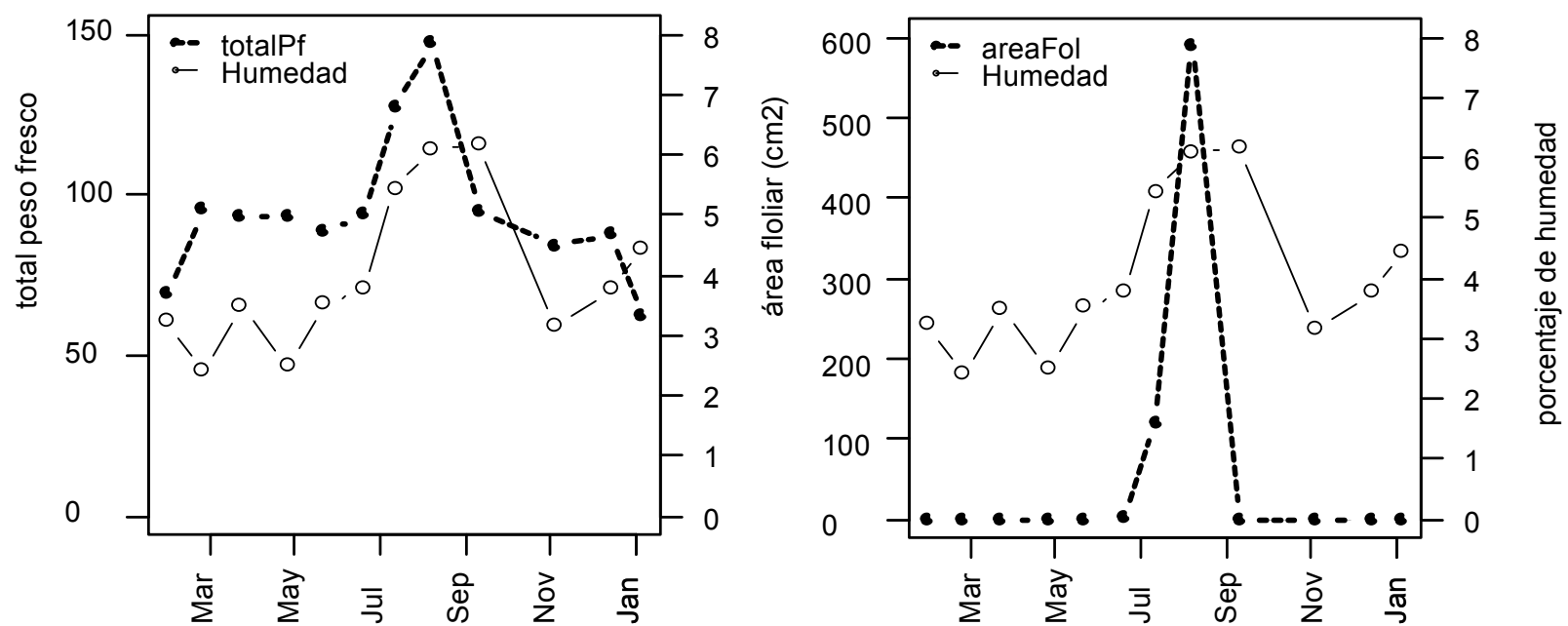

Figura 8. Relación directa entre el contenido de humedad del suelo y el peso fresco de la planta (izquierda) y con el área foliar (derecha) de Ismene amancaes.
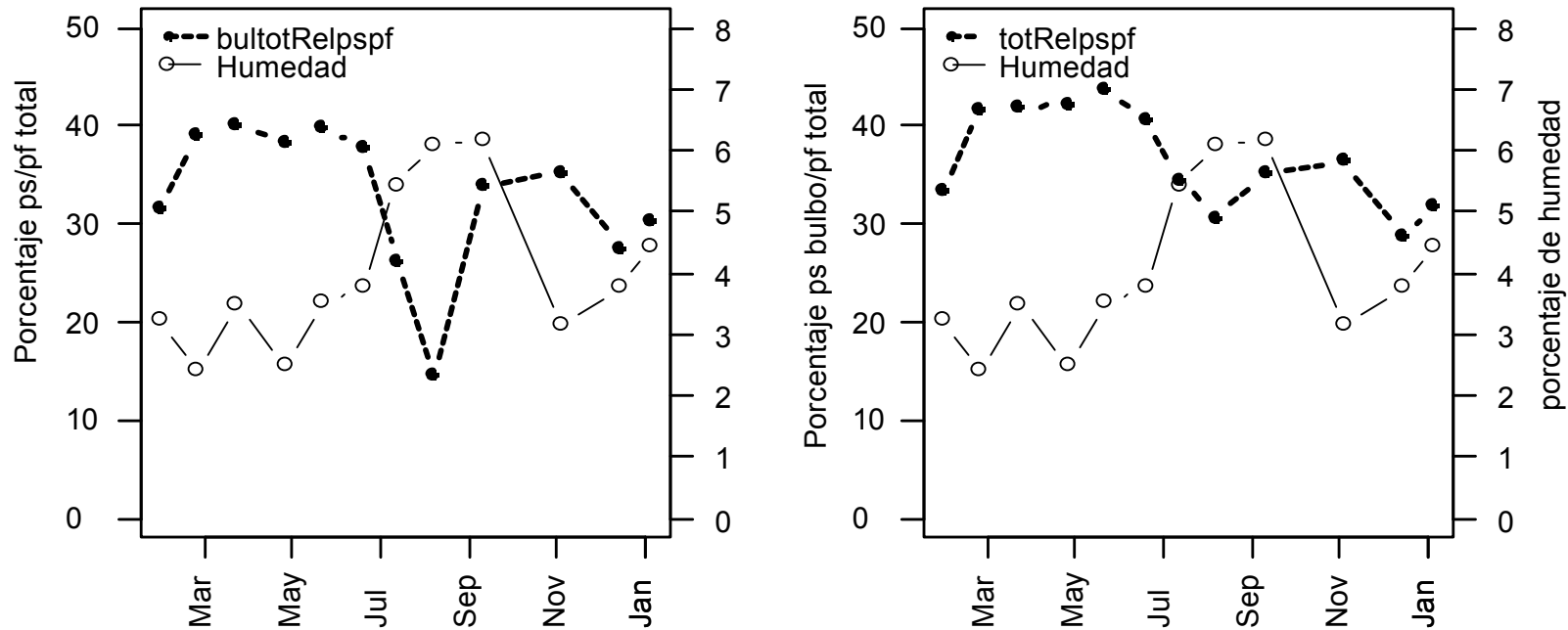

Figura 9. Relación inversa entre el contenido de humedad del suelo con el porcentaje de materia seca (ps) del bulbo y de la planta en relación a la biomasa total (pf) de Ismene amancaes. 
muy rápidamente alcanzando en el mes de mayo la altura del cuello del bulbo (Fig. 4) y en junio su emergencia (Fig. 5). El cuello del bulbo se constituye externamente por las vainas de las últimas hojas que emergieron el periodo anterior de Lomas y que formaban parte de la yema foliar que estuvo presente contigua a la inflorescencia en el periodo de mayo-junio. Seguido hacia el interior por las hojas (vainas y láminas) que se formaron luego, cuyas vainas tienen un arreglo concéntrico. Y más hacia el interior se presenta la hoja (sin vaina) adyacente a la inflorescencia, la inflorescencia y nueva yema foliar apical. La diferenciación de las yemas florales se inicia luego que se han formado las hojas que saldrán el siguiente año y en un periodo de máximo descenso de la humedad edáfica (noviembre). Llegan a constituir una pequeña inflorescencia que resulta ser la única ramificación que formará la yema apical que luego continuará formando hojitas. La yema foliar continuará su crecimiento pero en menor grado que la inflorescencia, la misma que en el periodo de Lomas emergerá primero. En julio sólo las primeras dos hojas de la yema foliar emergen y se pudo notar el gran grosor del escapo floral (Fig. 6) en este mes y que va disminuyendo hacia el periodo de fructificación para finalmente formar parte de los catafilos del bulbo.

Luego de la senescencia foliar (septiembre a noviembre), en el interior del bulbo sólo se observó la presencia de pequeñas hojas y hacia la parte central del disco basal, una yema foliar apical (Fig. 7). En diciembre se hace evidente la presencia de las pequeñas yemas florales rodeadas de pequeñas brácteas. Se observa también la yema foliar.

\section{Evaluación de las características del bulbo}

El contenido de humedad de la planta y del bulbo en peso fresco (pf) y peso seco (ps) y el área foliar tienen una relación directa con el contenido de humedad del suelo (Fig. 8), por lo que se puede señalar que el contenido de humedad del suelo influye positivamente en el desarrollo del área foliar y el contenido de humedad de toda la planta. Mientras que tiene una relación inversa con el porcentaje de materia seca del bulbo y de la planta (Fig. 9) lo cual refleja una alta absorción de agua que coincide con el máximo desarrollo de sus hojas (en agosto). Se observó asimismo que el grosor de los catafilos (que originalmente fueron las vainas de las hojas) variaba de 0,5 a 1,9 mm. Siendo el grosor de los catáfilos externos menor que los internos.

\section{Contenido de humedad del suelo}

A $20 \mathrm{~cm}$ de profundidad, nivel aproximado de ubicación del bulbo, los valores más bajos de contenido de humedad del suelo se presentaron de febrero a abril del 2004. En los meses siguientes se observó un incremento de la humedad hasta que en los meses de agosto y septiembre de obtuvieron los mayores valores para declinar precipitadamente en noviembre, periodo en que se considera se inicia la diferenciación de las yemas florales. Paralelamente se realizó a partir del mes de junio la evaluación del contenido de humedad del suelo a $5 \mathrm{~cm}$ de profundidad. A este nivel, el contenido de humedad del suelo está más sujeto a los cambios medio ambientales, este llega a tener en los meses húmedos mayores valores y en verano valores menores que a 20 $\mathrm{cm}$ de profundidad.

\section{Discusión}

Ismene amancaes presenta actividad a lo largo de todo el año. Al inicio del periodo no Lomas, cuando declina precipitadamente el contenido de humedad en el suelo (Agüero 2002) y se incrementa la temperatura (noviembre), ocurre el inicio de la diferenciación de las yemas florales. El desarrollo de la pequeńa inflorescencia dentro del bulbo, continua en los meses de verano, como se ha observado en algunos bulbos (Noy-Porat et al. 2009). Se encontró una relación inversa entre el contenido de humedad del suelo y la presencia de yemas florales. Esta es una etapa que depende de los recursos almacenados en el mismo bulbo (Boeken 1989). En años con incremento de temperatura en verano (evento El Niño) no se espera que se afecte el inicio de la diferenciación de las yemas florales, más bien por el retraso del periodo de humedad se prolonga este periodo de desarrollo dentro del bulbo, consumiendo reservas nutricias y disminuyendo la posibilidad de éxito reproductivo (Agüero \& Suni 1999). El desarrollo foliar en el periodo de Lomas presentó una relación directa con el contenido de humedad edáfica. Agüero y Suni (1999) reportaron un resultado semejante para plantas juveniles. El periodo de diferenciación y desarrollo de las estructuras reproductivas hasta la floración abarca ocho meses (noviembre a julio); mientras que la acumulación de fotoasimilados, los cuales son traslocados a la vaina de las hojas retornando e incrementando biomasa al bulbo, solamente dos meses (agosto, setiembre). Sin embargo este periodo es determinante para el éxito reproductivo del siguiente periodo.

\section{Agradecimientos}

Los autores expresan su profundo agradecimiento especialmente a PRODENA-AREQUIPA, CEMENTOS LIMA por el apoyo recibido, al Ingeniero M.Sc. Felipe Mendiburu por los análisis estadísticos de los datos con el programa estadístico R.

\section{Literatura citada}

Agüero S. \& M. Suni. 1999. Influencia del evento "El Niño 19971998" en el desarrollo de Ismene amancaes (Amaryllidaceae, Liliopsidae). En: El Niño 1997-98 y su Impacto sobre los Ecosistemas Marino y Terrestre. J. Tarazona y E. Castillo. (Eds.) Rev. peru. biol. Vol Extraordinario: 118-124.

Agüero S. 2002. Efecto de la humedad edáfica en el desarrollo y propagación de Ismene amancaes (R. \& P.) Herbert "amancaes" (Amaryllidaceae) en condiciones "insitu" y "ex situ”. Tesis para optar el título profesional de Biólogo, Universidad Nacional Mayor de San Marcos.

Boeken B. 1989. Life histories of desert geophytes - the demographic consequences of reproductive biomass partitioning patterns. Oecologia 80(2):278-283.

León B., A. Sagástegui, I. Sánchez, M. Zapata, A. Meerow \& A. Cano. 2006. Amaryllidaceae endémicas del Perú. En: El libro rojo de las plantas endémicas del Perú. Blanca León et al. (Eds.). Rev. peru. biol. Número especial 13(2):690s-697s

Noy-Porat T., M.A. Flaishman, A. Eshel, D. Sandler-Ziv \& R. Kamenetsky. 2009. Florogenesis of the Mediterranean geophyte Narcissus tazetta and temperature requirements for flower initiation and differentiation. Scientia Horticulturae. Sci. Hortic. 120(1):138-142. 
